\title{
Near-Field Spectral Effects due to Electromagnetic Surface Excitations
}

\author{
Andrei V. Shchegrov* \\ Rochester Theory Center for Optical Science and Engineering \\ and Department of Physics and Astronomy, University of Rochester, Rochester, New York 14627-0171
}

Karl Joulain, Rémi Carminati, and Jean-Jacques Greffet

Laboratoire d'Energétique Moléculaire et Macroscopique, Combustion, Ecole Centrale Paris, Centre National de la Recherche Scientifique, 92295, Châtenay-Malabry Cedex, France

(Received 14 March 2000)

\begin{abstract}
We demonstrate theoretically that the spectra of electromagnetic emission of surface systems can display remarkable differences in the near and the far zones. The spectral changes occur due to the loss of evanescent modes and are especially pronounced for systems which support surface waves.
\end{abstract}

PACS numbers: 78.20.-e, 05.40.-a, 44.40.+a, 87.64.Xx

Spectroscopy of electromagnetic radiation is perhaps the most powerful exploration tool employed in natural sciences: astronomy, atomic and molecular physics and chemistry, materials science, biology, etc. The central question considered in this paper-whether the spectral content of the radiation emitted by an object can change on propagation to the observer-usually does not arise, since it seems natural that nothing can happen to waves traveling through empty space. Surprisingly similar failure of common sense was put forward by the recent progress of near-field optical microscopy $[1,2]$, which achieves subwavelength resolution exactly because evanescent modes carrying subwavelength spatial information do not propagate far away from the object. However, a great deal of work devoted to such an irreversible change of spatial information on propagation has been accompanied with significantly lesser interest in the possibility of the change of spectral information.

The subject of spectral changes on propagation has, however, been addressed. In the 1980's, Wolf [3] predicted that the spectrum of light can be changed on propagation from the source to the observer, even through empty space. This effect, whose origin lies in the fluctuating nature of the source, has been intensively studied in a variety of systems [4,5]. Typically, the Wolf effect is manifested in small spectral shifts and can be viewed as a redistribution of the weights of different spectral components.

In this Letter, we demonstrate spectral changes, whose physical origin is very different from that of Wolf spectral shifts and lies in the presence of the evanescent component in the emitted field. We show that the near-field and far-field spectra of emitted electric fields can display drastic differences. For a broadband emission, such as thermal emission which is considered in detail in this paper, the near-field spectrum dominated by evanescent modes can be entirely different from the far-field spectrum of propagating modes. These spectral changes occur not due to the statistical nature of the source (as in the Wolf effect) but due to the loss of evanescent components on propagation. We analyze how such spectral changes can be enhanced by electromagnetic surface waves (SW) near the interface. These SW are known to play an important role in the enhancement of interaction between nanoparticles near the surface [6], in localization effects on random surfaces [7], in surface-enhanced Raman scattering [8], in extraordinary transmission of light through subwavelength holes [9], etc. In this Letter, we show that SW provide the leading contribution to the density of energy in the near-field zone of electromagnetic emission.

We now proceed with analyzing near-field effects in the spectra of thermal emission, which provides an easy way to excite both propagating and evanescent electromagnetic modes in a wide range of frequencies (at least, in the infrared [10-12]). Thermal emission is frequently associated with the textbook example of equilibrium blackbody radiation. The Planck spectrum $I_{B B}(\omega)$ of such radiation is obtained by multiplying the thermal energy $\theta(\omega, T)=\hbar \omega /\left[\exp \left(\hbar \omega / k_{B} T\right)-1\right]$ of a quantum oscillator by the density of oscillations (modes) per unit volume $N(\omega) d \omega=\omega^{2} d \omega /\left(\pi^{2} c^{3}\right)$ in the frequency interval $(\omega, \omega+d \omega)$, and dividing the result by $d \omega$ [13],

$$
I_{B B}(\omega)=\theta(\omega, T) N(\omega)=\frac{\hbar}{\pi^{2} c^{3}} \frac{\omega^{3}}{\exp \left(\hbar \omega / k_{B} T\right)-1} .
$$

Here $T$ is the body temperature, $k_{B}$ is Boltzmann's constants, $\hbar$ is Planck's constant divided by $2 \pi$, and $c$ is the speed of light in vacuum. A well-known representation of blackbody radiation is the equilibrium radiation in a closed cavity with lossy walls when only propagating modes of the field are taken into account.

To demonstrate the importance of near-field effects, we consider a somewhat more sophisticated example of thermal emission from a semi-infinite $(z<0)$ slab of homogeneous, nonmagnetic material held in local thermodynamic equilibrium at a uniform temperature $T$, into the empty halfspace $z>0$. We will describe the macroscopic dielectric properties of the material by a frequency-dependent, complex dielectric function $\varepsilon(\omega)=\varepsilon^{\prime}(\omega)+i \varepsilon^{\prime \prime}(\omega)$. The 
Fourier component $\mathbf{E}(\mathbf{r}, \omega)$ of the electric field $\mathbf{E}(\mathbf{r}, t)$ at a point $\mathbf{r}=(x, y, z)$ in the empty half space $z>0$ is generated by thermal currents with density $\mathbf{j}\left(\mathbf{r}^{\prime}, \omega\right)$, which is nonzero only for $z^{\prime}<0$. It can be computed following the procedure outlined in Refs. [12,14],

$E_{\alpha}(\mathbf{r}, \omega)=i \mu_{0} \omega \sum_{\beta=x, y, z} \int_{V} d^{3} r^{\prime} G_{\alpha \beta}\left(\mathbf{r}, \mathbf{r}^{\prime}, \omega\right) j_{\beta}\left(\mathbf{r}^{\prime}, \omega\right)$,

where $V$ is the volume of the hot body which occupies the half-space $z^{\prime}<0$, and $G_{\alpha \beta}\left(\mathbf{r}, \mathbf{r}^{\prime}, \omega\right)$ is the electromagnetic Green tensor for the system of two homogeneous materials separated by a planar interface $z=0$. According to the fluctuation-dissipation theorem [14], the fluctuations of thermal currents are described by the correlation function

$$
\begin{aligned}
\left\langle j_{\alpha}(\mathbf{r}, \omega) j_{\beta}\left(\mathbf{r}^{\prime}, \omega^{\prime}\right)\right\rangle= & \frac{\omega \theta(\omega, T)}{\pi} \varepsilon_{0} \varepsilon^{\prime \prime}(\omega) \delta_{\alpha \beta} \delta\left(\mathbf{r}-\mathbf{r}^{\prime}\right) \\
& \times \delta\left(\omega-\omega^{\prime}\right),
\end{aligned}
$$

where the angle brackets denote the statistical ensemble average. The Kronecker symbol $\delta_{\alpha \beta}$ and the spatial $\delta$ function in this formula follow from the assumption that the dielectric function is isotropic, homogeneous, and local [14].

The energy density $I(\mathbf{r}, \omega)$ of the emitted electric field at the point $\mathbf{r}$ is defined by the formula

$$
\sum_{\alpha=x, y, z} \frac{\varepsilon_{0}}{2}\left\langle E_{\alpha}^{*}(\mathbf{r}, \omega) E_{\alpha}\left(\mathbf{r}, \omega^{\prime}\right)\right\rangle \equiv I(\mathbf{r}, \omega) \delta\left(\omega-\omega^{\prime}\right) .
$$

Using Eqs. (2) and (3) into (4), we obtain, for $I(\mathbf{r}, \omega)$,

$$
\begin{aligned}
I(\mathbf{r}, \omega)= & 8 \pi^{3} \frac{\omega^{3}}{c^{4}} \theta(\omega, T) \varepsilon^{\prime \prime}(\omega) \sum_{\alpha, \beta=x, y, z} \int_{-\infty}^{0} d z^{\prime} \\
& \times \int \frac{d^{2} k_{\|}}{(2 \pi)^{2}}\left|g_{\alpha \beta}\left(\mathbf{k}_{\|}, \omega \mid z, z^{\prime}\right)\right|^{2}
\end{aligned}
$$

where $g_{\alpha \beta}\left(\mathbf{k}_{\|}, \omega \mid z, z^{\prime}\right)$ is the analytically known [12] 2D spatial Fourier transform (in $x$ and $y$ ) of the Green's tensor $G_{\alpha \beta}\left(\mathbf{r}, \mathbf{r}^{\prime}, \omega\right)$. Note that $I(\mathbf{r}, \omega)$ in Eq. (5) is independent of $x$ and $y$, due to the translational invariance of the system in $x$ and $y$ directions.

We now assume that the interface $z=0$ between the material and a vacuum can support electromagnetic SW. The dispersion relation between the wave number $k_{\|}=$ $\left|\mathbf{k}_{\|}\right|$and frequency $\omega$ of $\mathrm{SW}$ is

$$
\left[k_{\|}^{\mathrm{SW}}(\omega)\right]^{2}=\left(\omega^{2} / c^{2}\right) \varepsilon(\omega) /[\varepsilon(\omega)+1] .
$$

Such waves exist for materials having $\varepsilon^{\prime}(\omega)<-1$ in one or several frequency ranges [10]. We consider $\mathrm{SiC}$, which supports SW known as surface phonon polaritons and which has been used in previous experimental [11] and theoretical [12] investigations of thermal emission. The dielectric function of this material is given by the expression $\varepsilon(\omega)=\varepsilon_{\infty}\left(\omega_{L}^{2}-\omega^{2}-i \gamma \omega\right) /\left(\omega_{T}^{2}-\omega^{2}-i \gamma \omega\right)$ with $\varepsilon_{\infty}=6.7, \quad \omega_{L}=182.7 \times 10^{12} \mathrm{~s}^{-1}, \quad \omega_{T}=149.5 \times$ $10^{12} \mathrm{~s}^{-1}$, and $\gamma=0.9 \times 10^{12} \mathrm{~s}^{-1}$ [11]. By substituting $\varepsilon(\omega)$ into Eq. (5) and performing a straightforward evaluation of integrals (only the integral over the magnitude of $\mathbf{k}_{\|}$has to be calculated numerically, the other two integrals can be evaluated analytically), we obtain the spectra of thermal emission for $\mathrm{SiC}$ at different heights $z$ above the surface. We plot the results in Fig. 1 in the frequency range $0<\omega<400 \times 10^{12} \mathrm{~s}^{-1}$ for $T=300 \mathrm{~K}$ at three different heights.

Although one could expect to find differences of the $\mathrm{SiC}$ spectra with the blackbody spectrum (1), it is striking that near-field and far-field spectra of the same $\mathrm{SiC}$ sample are so dramatically different, as seen in Fig. 1. An observer doing a traditional far-zone spectroscopic measurement (Fig. 1a) would detect the spectrum with a rather wide dip in the range $150 \times 10^{12} \mathrm{~s}^{-1}<\omega<180 \times 10^{12} \mathrm{~s}^{-1}$ due to the low emissivity of $\mathrm{SiC}$ in that range [11]. Note that the sample effectively acts as a nonradiating source in this frequency range. However, when the probe moves within a subwavelength distance from the material (typical thermal emission wavelengths at $T=300 \mathrm{~K}$ are of the order of $10 \mu \mathrm{m}$ ), the spectrum starts to change rapidly. In Fig. $1 \mathrm{~b}$, showing the emission spectrum at $2 \mu \mathrm{m}$ above the surface, this change is seen as a peak emerging at $\omega=178.7 \times 10^{12} \mathrm{~s}^{-1}$. At very close distances (Fig. 1c), the peak becomes so strong that an observer would surprisingly see almost monochromatic emission with photon energies not represented in the far zone.

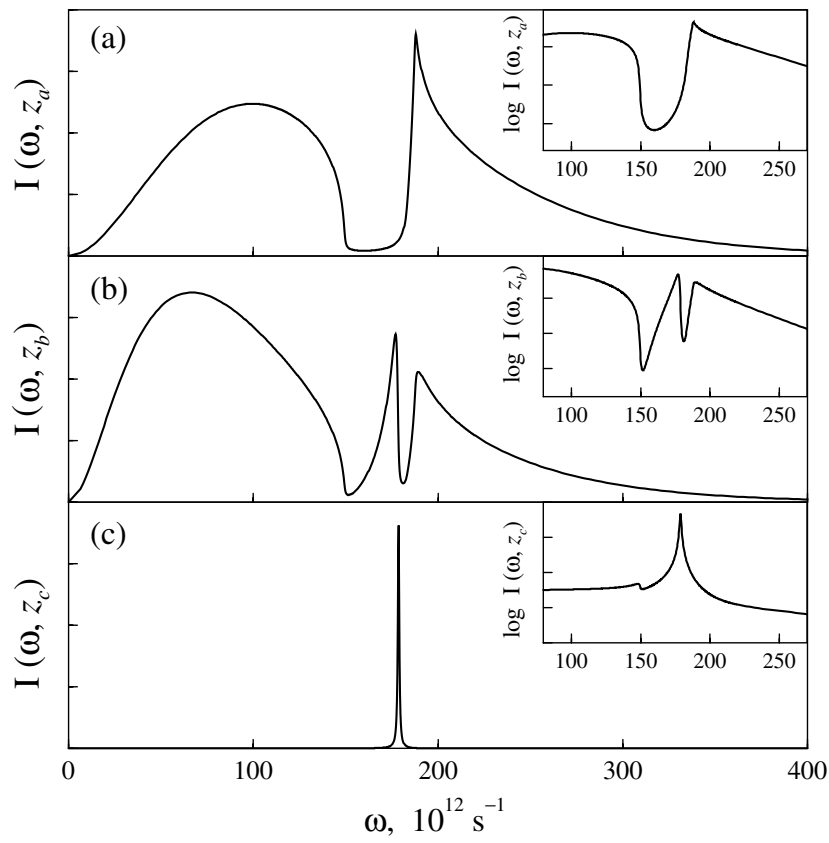

FIG. 1. Spectra of thermal emission of a semi-infinite sample of $\mathrm{SiC}$ at $T=300 \mathrm{~K}$ and three different heights above the surface: (a) $z_{a}=1000 \mu \mathrm{m}$, (b) $z_{b}=2 \mu \mathrm{m}$, (c) $z_{c}=0.1 \mu \mathrm{m}$. The insets magnify the spectra plotted on a semilog scale in the range of strong contribution from evanescent surface modes. 
Thus, we find that the spectrum changes qualitatively on propagation. The occurrence of such striking spectral changes is related to SW existing in the region $150 \times$ $10^{12} \mathrm{~s}^{-1}<\omega<180 \times 10^{12} \mathrm{~s}^{-1}$. We shall now clarify the mechanism of the formation of spectra $I(\mathbf{r}, \omega)$ at different distances from the surface.

We note that the spectrum of the elctric field (5) has a similar structure as that of blackbody radiation (1),

$$
I(z, \omega)=\theta(\omega, T) N(z, \omega) .
$$

In Eq. (1), $N(\mathbf{r}, \omega)$ does not depend on $\mathbf{r}$, while in Eq. (5) it depends on $z$. Equation (7) is a pivotal point of our paper since it accounts for the evolution of the spectrum on propagation by relating it to the local density of electromagnetic modes $N(\mathbf{r}, \omega)$. Note that $N(\mathbf{r}, \omega)$ includes only relevant modes excited in the material $(z<0)$ and emitted into vacuum $(z>0)$. The correct counting of modes is done automatically in the integral in Eq. (5), which has a typical structure that relates the density of modes to the Green's tensor of the system. The function $\left|g_{\alpha \beta}\left(\mathbf{k}_{\|}, \omega \mid z, z^{\prime}\right)\right|^{2}$ in Eq. (5) represents a mode that is excited in the plane $z^{\prime}$ with a $2 \mathrm{D}$ wave vector $\mathbf{k}_{\|}$and polarization $\beta$ and arrives at the plane $z$ with the same 2D wave vector (due to translational invariance in $x$ and $y$ ) and polarization $\alpha$. The sum over $\beta$ and the integrals over $\mathbf{k}_{\|}$ and $z^{\prime}<0$ take into account all possible modes that are initially excited.

The origin of a sharp peak seen in the near-field emission spectrum (Fig. 1c) becomes clear when we analyze the dispersion relation (6) for SW (Fig. 2). Near the frequency $\omega_{\max }$ defined by the condition $\varepsilon^{\prime}\left(\omega_{\max }\right)=-1$, there exists a large number of surface modes with different wave numbers but with frequencies that are very close to each other. Therefore, the density of surface modes will necessarily display a strong peak at $\omega=\omega_{\max }$. However, since SW decay exponentially away from the surface, this peak is not seen in the far zone (Fig. 1a).

To achieve a detailed understanding of the $z$ dependence of the emission spectrum, we calculate an approximate expression for the density of modes $N(z, \omega)$ from Eqs. (5) and (7) for small distances $z$ and in the limit of large $k_{\|}$

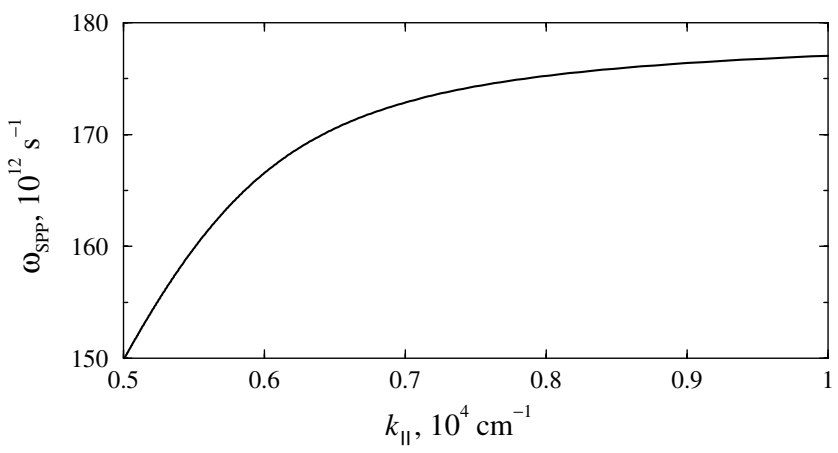

FIG. 2. Dispersion curve for $\mathrm{SW}$ on the vacuum-SiC planar interface. $\operatorname{Re}\left(\omega_{S P P}\right)$ is calculated for a given real $k_{\|}$. (according to Fig. 2, the modes with large $k_{\|}$define the behavior near the peak):

$$
N(z, \omega)=\frac{\varepsilon^{\prime \prime}(\omega)}{|1+\varepsilon(\omega)|^{2}} \frac{1}{16 \pi^{2} \omega z^{3}} .
$$

This $1 / z^{3}$ contribution can be recognized as a well-known quasistatic behavior exhibited near the surfaces of all materials $[12,14]$. Note that if $\varepsilon^{\prime \prime}$ is not very large at $\omega=\omega_{\max }$ then the density of modes exhibits a resonance at that frequency. This is the origin of the peak in the near-field spectrum of $\mathrm{SiC}$ at $\omega=178.7 \times 10^{12} \mathrm{~s}^{-1}$. The presence of a resonance in the density of modes $N(z, \omega)$ is, however, not required for observing spectral changes caused by the loss of evanescent modes. Indeed, in the short distance regime, the spectrum is given by Eq. (8), whereas, in the far field, the spectrum is given by Eq. (1) multiplied by the emissivity of the surface. Thus, even in the absence of resonant SW, the near-field spectrum is different from the far-field spectrum, but the changes are less dramatic.

The result (8) is valid only in the limit of distances much smaller than the wavelength. We show in Fig. 3 the variation of the spectral density $I(\omega, z)$ with the distance $z$ from the surface. We consider two different frequencies none of which is very close to the resonance at $\omega_{\max }$. In agreement with Eq. (8), we observe that the spectral density increases sharply for $z<1 \mu \mathrm{m}$, i.e., when the distance to the surface is much smaller than the wavelength. However, the decay behavior of the two curves for larger values of $z$ exhibits an essential difference. The exponential decay seen in the solid curve for the values of $z$ between 1 and $5 \mu \mathrm{m}$ is a signature of the presence of a SW whose energy decays exponentially with $z$. This SW is a surface phonon polariton that exists at $\omega=166 \times 10^{12} \mathrm{~s}^{-1}$ (solid curve) but not at $\omega=210 \times 10^{12} \mathrm{~s}^{-1}$ (dashed curve). The different $z$ dependence of the $I(\omega, z)$ for different values of $\omega$ causes the spectrum to change on propagation of emitted radiation from the surface to the far zone.

This analysis allows us to conclude that the spectral changes in thermal emission should be observable in a wide variety of solid-state systems supporting evanescent

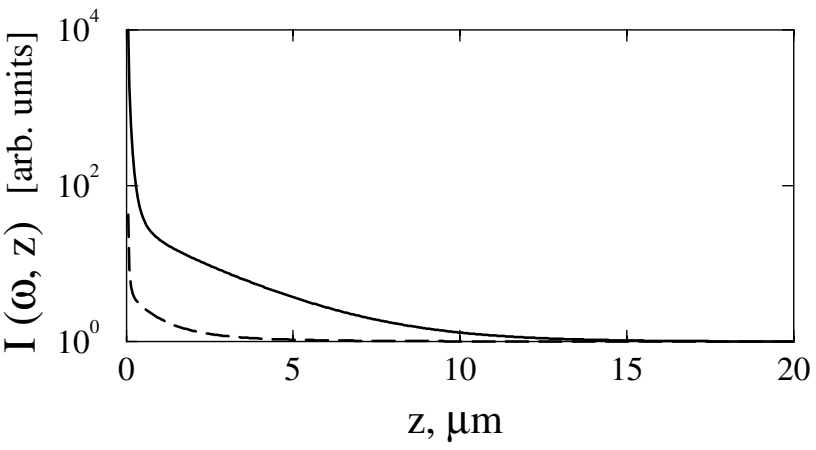

FIG. 3. The variation of the spectral density of the thermal emission for $\mathrm{SiC}$ at $T=300 \mathrm{~K}$ with the distance $z$ from observer to the surface. Solid line: $\omega=166 \times 10^{12} \mathrm{~s}^{-1}(\lambda=$ $11.4 \mu \mathrm{m})$, dashed line: $\omega=210 \times 10^{12} \mathrm{~s}^{-1}(\lambda=9.0 \mu \mathrm{m})$. 
surface waves or guided waves (in layered structures [5]). Resonant features in the near-field spectra (such as in Fig. 1c) correspond to resonances in the local density of surface modes $N(z, \omega)$ and appear when the dispersion curve for SW has a flat portion (as in Fig. 2). In addition to our example of $\mathrm{SiC}$, such resonances $N(z, \omega)$ are displayed by metals (supporting surface plasmon polaritons), semiconductors (supporting surface exciton polaritons $\Phi$ ), and several other materials. Yet, a peak in the emission intensity $I(z, \omega)$ will be observable only if $\theta(\omega, T)$ is not too small. For example, the near-field spectrum of thermal emission from amorphous glass near-field spectrum has a sharp peak for $\omega=9.24 \times 10^{13} \mathrm{~s}^{-1}(\lambda=20.4 \mu \mathrm{m})$ visible at room temperature. All of the III-V and II-VI semiconductors can support surface waves in the midinfrared. However, although the number of modes (8) has a resonance in the case of silver at about $\omega=5.57 \times 10^{15} \mathrm{~s}^{-1}$ $(\lambda=0.339 \mu \mathrm{m})$, no sharp peak is seen if the temperature silver sample is lower than $4000 \mathrm{~K}$.

Equations (7) and (8) also suggest a new application for near-field spectroscopy. As a near-field spectrum at a given distance to the interface gives access to $\varepsilon^{\prime \prime}(\omega) / \mid 1+$ $\left.\varepsilon(\omega)\right|^{2}$, one can hope to retrieve the material dielectric constant, similar to the method usually used to obtain $\varepsilon$ from reflectivity measurements [15]. With the rapid development of near-field optical microscopy, such near-field spectra can be measured. This could open the way to a new technique of local solid-state spectroscopy. Finally, we anticipate that the effects reported in this paper should significantly improve our understanding of the radiative heat transfer at nanometric scale with particular applications in the field of near-field microscopies. This might have applications for high density storage where the local control of temperature is essential in the writing process. Also, note that the effect of near-field thermal fluctuations was measured recently using the induced brownian motion on an atomic force microscope tip [16].

To summarize, we have demonstrated that the spectrum of thermal emission can undergo significant, qualitative changes on propagation due to the loss of evanescent modes. Such novel spectral changes are caused by the change in the local density of emitted electromagnetic modes, and are especially pronounced in the systems supporting surface waves.

We thank Professor Emil Wolf and Dr. Carsten Henkel for useful discussions. The work of A. V.S. was supported by the Engineering Research Program of the Office of Basic Energy Science at the Department of Energy under Grant No. DE-FG02-90ER 14119 and by the Air Force Office of Scientific Research under Grant No. F4962000-1-0125.

*Present address: KLA-Tencor Corporation, 160 Rio Robles, San Jose, CA 95134.

Email address: Andrei.Shchegrov@KLA-Tencor.com

[1] Near Field Optics, edited by D. W. Pohl and D. Courjon (Kluwer, Dordrecht, 1993); J.-J. Greffet and R. Carminati, Prog. Surf. Sci. 56, 133 (1997).

[2] Q. Wu, R. D. Grober, D. Gammon, and D. S. Katzer, Phys. Rev. Lett. 83, 2652 (1999).

[3] E. Wolf, Phys. Rev. Lett. 56, 1370 (1986); Nature (London) 326, 363 (1987).

[4] E. Wolf and D. F. V. James, Rep. Prog. Phys. 59, 771 (1996).

[5] T. A. Leskova, A. A. Maradudin, A. V. Shchegrov, and E. R. Méndez, Phys. Rev. Lett. 79, 1010 (1997); A. V. Shchegrov and A. A. Maradudin, Phys. Rev. B 59, 7732 (1999).

[6] H. R. Stuart and D. G. Hall, Phys. Rev. Lett. 80, 5663 (1998).

[7] A. R. McGurn, A. A. Maradudin, and V. Celli, Phys. Rev. B 31, 4866 (1985); S. I. Bozhevolnyi, I. I. Smolyaninov, and A. V. Zayats, Phys. Rev. B 51, 17916 (1995).

[8] Surface-Enhanced Raman Scattering, edited by R.K. Chang and T.E. Furtak (Plenum, New York, 1982).

[9] T. W. Ebbesen, H. J. Lezec, H. F. Ghaemi, T. Thio, and P. A. Wolff, Nature (London) 391, 667 (1998).

[10] E. A. Vinogradov, G. N. Zhizhin, and V. I. Yudson, in Surface Polaritons, edited by V. M. Agranovich and D. L. Mills (North-Holland, Amsterdam, 1982), p. 145.

[11] P. J. Hesketh, J. N. Zemel, and B. Gebhart, Nature (London) 324, 549 (1986); J. Le Gall, M. Olivier, and J.-J. Greffet, Phys. Rev. B 55, 10105 (1997).

[12] R. Carminati and J.-J. Greffet, Phys. Rev. Lett. 82, 1660 (1999).

[13] L. D. Landau, E. M. Lifshitz, and L. P. Pitaevskii, Statistical Physics, Part I (Pergamon, Oxford, 1980), p. 183.

[14] S. M. Rytov, Yu. A. Kravtsov, and V. I. Tatarskii, Principles of Statistical Radiophysics 3 (Springer-Verlag, Berlin, 1989), p. 118.

[15] E. W. Palik, Handbook of Optical Constants of Solids (Academic Press, San Diego, 1985).

[16] I. Dorofeyev, H. Fuchs, G. Wenning, and B. Gotsmann, Phys. Rev. Lett. 83, 2402 (1999). 